\title{
The effects of set volume during isolated lumbar extension resistance training in recreationally trained males
}

James Steele, Adam Fitzpatrick, Stewart S Bruce-Low, James P Fisher

Background/Aim: Exercise designed to condition the lumbar extensor musculature is often included in resistance training (RT) programs. It is suggested that deconditioning of this musculature may be linked to low back pain. Thus effective means of conditioning these muscles are of interest to pursue. Evidence suggests that isolated lumbar extension (ILEX) RT might be most efficacious, however, the best means of manipulating resistance training variables in this regard is unknown. Set volume is an easily manipulated RT variable and one thought to also interact with training status. As such this study sought to examine the effect of set volume during ILEX RT in trained males. Methods: Trained males previously engaged in exercises designed to condition the lumbar extensors underwent a 6 week intervention and were randomised to either a single set (1ST, $n=9$ ), multiple set (3ST, $n=$ 8 ) or control group (CON, $n=9$ ). Pre and post testing of ILEX strength was conducted. Results: Both 1ST and 3ST significantly increased ILEX strength $(p<0.05)$ with large effect sizes ( $d=0.89$ and 0.95 respectively) whereas the CON group produced significant losses $(-8.9 \%)$ with a moderate effect size $(d=-0.53)$. There was no statistically significant difference in ILEX strength gains when 1ST and 3ST were directly compared ( $p=0.336)$. Conclusions: The results of this study suggest that significant ILEX strength changes occur in trained males as a result of 6 weeks of ILEX RT and that these changes are unaffected by set volume. 
2 James Steele ${ }^{1}$, Adam Fitzpatrick ${ }^{1}$, Stewart Bruce-Low ${ }^{1}$, James Fisher ${ }^{1}$

$3{ }^{1}$ Centre for Health, Exercise and Sport Science, Southampton Solent University, Southampton,

4 Hampshire, UK

5

6 Contact Author: James Steele

7 Centre for Health, Exercise and Sport Science,

8 Southampton Solent University,

9 East Park Terrace,

10 Southampton,

11 Hampshire,

12 United Kingdom

$13 \mathrm{SO} 532 \mathrm{HE}$

14 Email: james.steele@solent.ac.uk

15

16

17

18

19

20

21

22

23

24

25 


\section{Introduction}

Low back pain (LBP) remains a highly prevalent condition in both general (WHO, 1998; ONS, 2000; ONS, 2010; Waddell \& Burton, 2000; Walker, 2000; NICE, 2009) and athletic populations (Graned \& Morelli, 1988; Sward et al., 1990; Kraft, 2002; Bono, 2004; Bahr et al., 2004; Quinney, 1997;

31 Haykowsky, 1999). The use of resistance training (RT) to reduce injury risk has been suggested (Stone, 1990) and a systematic review has recently reported it to reduce sporting injury risk by one third

33 (Lauersen et al., 2013). As evidence seems to suggest that deconditioning of the lumbar extensor 34 musculature (lumbar erector spinae, multifidus, and quadratus lumborum) may be a factor associated with LBP and increased injury risk (Steele et al., 2014 ${ }^{\mathrm{a}}$ ) research has attempted to evaluate the best approaches for conditioning this musculature through RT.

Mayer et al. (2008) have suggested differing exercise approaches to condition the lumbar extensors which have recently been reviewed with evidence suggesting that, due to the restraint system preventing rotation of the pelvis, isolated lumbar extension (ILEX) exercise devices may be optimal (Steele et al., 2013). However, when considering an RT programme, variables such as load, effort, repetitions, repetition duration, volume, frequency, etc. should also be considered (American College of Sports Medicine, 2009; Fisher et al., 2011). The manipulation of such variables when employing ILEX has been reviewed when considering patients suffering with chronic LBP for outcomes such as pain and disability (Steele et al., 2014 ), but does not exist for asymptomatic people who may be interested in injury prevention.

However, a number of studies have considered asymptomatic individuals, and the effect of RT variable manipulation upon ILEX strength as an outcome examining the volume, frequency, specificity and range of motion during training, considering both previously untrained participants (Graves et al., 1990a; Carpenter et al., 1991; Graves et al., 1992; Graves et al., 1991) as well as people with RT 
50 experience (Tucci et al., 1992). To date there appears only one study (Graves et al., 1991) which has 51 considered training volume using ILEX but again this was on untrained participants. This research

52 considered participants performing either one or two sets of dynamic and/or isometric ILEX training 53 reporting no differences (Graves et al., 1991). Set volume in RT has historically been a contentious issue 54 with recommendations supporting multiple set protocols (American College of Sports Medicine, 2009) 55 being supported by meta-analyses (Rhea et al., 2003; Peterson et al., 2004; Wolfe et al., 2004). Others 56 have however critiqued these meta-analyses (Winett, 2004; Otto \& Carpinelli, 2006). As the body of 57 literature has progressed, further reviews and meta-analyses have offered support, for multiple (Krieger, 58 2009; Frohlich et al., 2010), or single set protocols (Fisher et al., 2011; Carpinelli, 2012) for strength.

More recently further empirical research has examined set volume within RT with some support for multiple set approaches (Marshall et al., 2011; Radaelli et al., 2014 ), and other studies finding no differences between single and multiple set routines (Radaelli et al., 2014c; 2013ª $2013^{\mathrm{b}}$; Kadir et al., 2014; Adnan et al., 2014; Correa et al., 2014; Baker et al., 2013).

Differentiating between trained and previously untrained participants within the research appears to be an important consideration as it is suggested that the level of trainability affects the interaction of specific RT variable manipulation with outcomes, particularly with respect to set volume (Frohlich et al., 2010). In addition, that trained people should perform a larger training volume than untrained people (American College of Sports Medicine, 2009). Though Graves et al. (1991) reported no difference between one and two sets of ILEX the authors only considered untrained participants and did not consider the effects of greater set volumes typically suggested as being optimal for strength $(\geq 3$ sets; Krieger, 2009). Since RT is shown to be an effective intervention in trained and sporting populations for reducing injury risk it is of interest to investigate the effects of set volume during ILEX exercise in a trained population looking specifically at the lumbar extensor muscles. As such the aim of the present study was to consider the effects of single and multiple set ILEX exercise in trained males. 


\section{Materials \& Methods}

\section{$76 \quad$ Study Design}

A randomised controlled trial was adopted with three experimental groups examining the effects of set volume upon ILEX strength in recreationally trained participants. The study design was approved

79 in writing by the relevant ethics committee at the author's institution.

\section{$81 \quad$ Participants}

Participants were required to be males between 18-30 years old, have been involved in a RT programme for a minimum of 6 months at a minimum frequency of $2 \mathrm{x} /$ week and including participation in exercises designed to condition the lumbar extensors (Mayer et al., 2008; Steele et al., 2013) at the beginning of the study. Furthermore, participants were to have no medical condition for which RT is contraindicated to participate. Specific exclusion criteria included, 1) history of LBP or lumbar spine pathologies or known deformities, 2) currently experiencing LBP, 3) knee or hip disorders contraindicating use of the ILEX device, 4) inability or unwillingness to cease participation in other lumbar conditioning exercises for the duration of the study, 5) currently taking any illegal ergogenic aids or nutritional supplementation. Written informed consent was obtained from all participants.

Power analysis of research using ILEX (Graves et al., 1990a) was conducted to determine participant numbers (n) using an effect size (ES), calculated using Cohen's $d$ (Cohen, 1992), of 1.26 for the improvements in ILEX strength. Participant numbers were calculated using equations from Whitley and Ball (2002) revealing each group required 10 participants to meet required power of $\beta=0.8$ at an alpha value of $p \leq 0.05$.

Thirty participants were initially identified and recruited. Figure 1 shows a CONSORT diagram highlighting participant numbers for enrolment, allocation, follow-up and analysis stages. No initial 
98 dropouts were recorded after eligibility assessment so 30 participants were randomised using a random 99 drawing of cards to one of three groups; a single set group (1ST; $n=10)$, a multiple set group (3ST; $n=$ 100 10), and a non-training control group $(\mathrm{CON} ; \mathrm{n}=10)$.

101

102 Equipment

103 Stature was measured using a stadiometer (Holtan ltd, Crymych, Dyfed), body mass measured using 104 scales (SECA, Germany) and Body Mass Index (BMI) calculated. Isometric ILEX strength testing, range

105 of motion and training were performed using an ILEX device (MedX, Ocala, Florida; Figure 2). The 106 device has been shown reliable in assessing isometric strength at repeated angles in asymptomatic $(\mathrm{r}=$

1070.81 to 0.97 ; Graves et al., $\left.1990^{b}\right)$ and symptomatic participants ( $r=0.57$ to 0.93 ; Robinson et al., 1992),

108 and valid in measurement (Pollock et al., 1991; Inanami, 1991).

109

$110 \quad$ Participant Testing

111 ILEX strength was tested twice, on separate days (at least 72 hours apart to avoid residual fatigue or 112 soreness) before with the first acting as a familiarisation, and once after the RT intervention. Each test

113 using the ILEX device involved maximal voluntary isometric contractions at various angles through the

114 participant's full range of motion. Details of the full test protocol using the MedX and its restraint

115 mechanisms have been documented elsewhere (Graves et al., 1990 ). ILEX strength averaged across the

116 participant's full range of motion was considered.

117

\section{$118 \quad$ Participant Training}

119 Training was conducted at a frequency of $1 \mathrm{x} /$ week for a period of 6 weeks. This frequency of training 120 has been shown to significantly improve ILEX strength and was chosen over more frequent training

121 (Bruce-Low et al., 2012) due to potential for overtraining when the lumbar extensor muscles are isolated 
122 (Graves et al., 1990a). Both groups performed one set of variable resistance ILEX exercise. Both

123 performed a warm-up set lasting 60 seconds using $50 \%$ of their training load. Starting training load was

$12450 \%$ of max recorded tested functional torque (TFT) during maximal isometric testing for both groups

125 and repetitions performed until momentary muscular failure (MMF) in order to control for effort (Steele,

126 2014). Repetitions were performed taking at least 2 seconds to complete the concentric phase, holding

127 for 1 second in full extension and taking at least 4 seconds for the eccentric phase. The 3ST group rested

128 for 3 minutes between each set during which time participants remained seated in the ILEX device with

129 the restraints loosened. Resistance load was increased by $10 \%$ in the next session or set once the

130 participant was able to perform more than 12 repetitions using their current load before achieving MMF.

131 However, in order to maintain a similar repetition range for each set in the 3ST group, load was reduced

132 by $5 \%$ in the next set if the participant was unable to complete at least 8 repetitions before achieving

133 MMF (Medeiros et al., 2013). All groups were asked to refrain from using any other lumbar conditioning

134 exercises for the duration of the study.

136 Data Analysis

137 Participants that missed 2 or more sessions were excluded from analysis. Twenty six participants' (1ST $138 \mathrm{n}=9 ; 3 \mathrm{ST} \mathrm{n}=8 ; \mathrm{CON} \mathrm{n}=9$ ) data were available for analysis. Assumptions of normality of distribution 139 were examined using a Kolomogorov-Smirnov test. As not all data sets met assumptions of normality of 140 distribution non-parametric analyses were performed. Demographic characteristics, baseline ILEX 141 strength and the effects of the group (independent variable) upon the absolute change in ILEX strength 142 (dependent variable) was examined using a Kruskal-Wallis one way analysis of variance. Significant 143 results from the Kruskal-Wallis test were further subjected to post hoc planned comparisons using Mann144 Whitney U tests between $1 \mathrm{ST}$ and 3ST. Further, 95\% confidence intervals (CI) were calculated in 145 addition to ES using Cohen's $d$ (Cohen, 1992) for each outcome to examine significance and magnitude 
146 of effects within groups where significant changes were considered where 95\%CIs did not cross zero 147 and for magnitudes an ES of $0.20-0.49$ was considered as small, $0.50-0.79$ as moderate and $\geq 0.80$ as 148 large. Statistical analysis was performed using SPSS (IBM Statistics for Windows, Version 20.0; IBM, 149 Portsmouth, Hampshire, UK) and $p \leq 0.05$ set as the limit for statistical significance.

\section{Results \& Discussion}

\section{$152 \quad$ Participant Demographics}

153 Participant demographics are shown in Table 1. There were no significant between group effects for any 154 demographic characteristics.

\section{Strength}

157 Figure 2 shows mean changes and 95\%CIs for ILEX strength for each group. The Kruskal-Wallis one 158 way analysis of variance revealed a significant between groups effect for ILEX strength $\left(X^{2}(2)=11.645\right.$, $159 p=0.003$; mean rank for $1 \mathrm{ST}=16.22,3 \mathrm{ST}=18.25, \mathrm{CON}=6.56)$. Planned comparison between $1 \mathrm{ST}$ 160 and 3ST using Mann-Whitney U revealed no significant difference for ILEX strength $(21.00 U=26.000$, $161 p=0.336$; Median \pm Interquartile Range, $28.48 \pm 43.49$ and $46.30 \pm 40.58$ respectively). $95 \%$ CIs indicated 162 that both 1ST and 3ST groups significantly increased strength ( $8.31 \%$ and $10.68 \%$ respectively) with 163 ESs considered large (0.89 and 0.95 respectively) whereas there was a significant reduction in ILEX 164 strength in the control group (-8.9\%) with a moderate ES (-0.53).

\section{$\underline{\text { Discussion }}$}

167 This study examined the effects of set volume in recreationally trained male participants performing an 168 ILEX RT intervention. The results indicated that both 1ST and 3ST significantly improved their ILEX 169 strength with similarly large ESs, whereas the CON group lost strength over the intervention period. No 
170 differences between 1ST and 3ST for ILEX strength change were found as a result of planned 171 comparisons.

172

173 Reviews and meta-analyses offer divergent conclusions regarding the effects of set volume (Fisher et al.,

174 2011; Otto \& Carpinelli, 2006; Rhea et al., 2003; Peterson et al., 2004; Wolfe et al., 2004; Winett, 2004;

175 Krieger et al., 2009; Frohlich et al., 2010; Carpinelli, 2012). Within this study, and in contrast to earlier

176 research by Graves et al. (1991) examining set volume and ILEX RT, we used trained participants as it

177 is believed that training status might interact with the effects of set volume (Frohlich et al., 2010). In

178 particular it is recommended that greater set volumes be utilised by trained people (American College of

179 Sports Medicine, 2009). The results of this study seem to suggest that, at least for ILEX training, set

180 volume does not impact upon strength changes in trained people. This finding is in agreement with the

181 majority of more recent empirical research regarding set volume indicating multiple sets appear to offer

182 similar efficacy upon strength gains to single set approaches both in untrained (Radaelli et al., 2014 ${ }^{\text {b; }}$

183 2014 ; 2013 ${ }^{\mathrm{a}} ; 2013^{\mathrm{b}}$; Kadir et al., 2014) and recreationally trained participants (Adnan et al., 2014; Baker

184 et al., 2013). Research by Marshall et al. (2011) in trained males does however contrast this. Though

185 they reported no difference between one and four sets they noted significantly greater strength changes

186 with eight sets. Though this may have been due to this group containing a high proportion of

187 medium/high responders (Carpinelli, 2012) it remains a possibility that a higher set volume than that

188 used in the present study ( $\geq 3$ sets) may induce greater ILEX strength gains. Indeed another recent study

189 by Radaelli et al. (2014 $)$ suggests a dose response relationship between 1, 3 and 5 sets in untrained yet

190 active males. However, it should be noted that the lumbar extensors have been suggested to be

191 particularly prone to overtraining (Graves et al., 1990a). All participants within the 3ST group reported

192 feeling nauseous and entirely exhausted (not limited to the lumbar extensors) during training whereas

193 only one of the 1ST group displayed these symptoms. Hass et al. (2000) reported that dropout rate was 
194 higher for a multiple set RT program compared with one employing single set. Twenty five percent 195 dropped out from the multiple set group ( 5 for lack of adherence and 2 for injuries) compared with none 196 in the single set group. Though no injuries were reported from our participants due to the training, the 197 potential of increased injury risk and impact of higher volume high effort training upon adherence, in 198 addition to the increased time required, should be weighed against any potential for greater gains with 199 much higher set volumes.

201 It is worth discussing that ILEX may be unique even when considering trained people. As noted, the 202 participants in this study were previously participating in RT involving exercises suggested by Mayer et 203 al. (2008) to condition the lumbar extensors (including deadlifts, good mornings, trunk extension 204 machines etc.). However, though two participants had previously used the ILEX device used in this study 205 (though the specific duration or implementation of this was not known), not all participants had been 206 previously involved in ILEX training specifically. Pollock et al. (1989) also reported considerable 207 improvements in average ILEX strength $(\sim 37 \%)$ in trained individuals (at least 1 year training 208 experience) engaged in similar exercises prior to ILEX RT interventions (including use of a 209 commercially available trunk extension resistance machine). Pollock et al. (1989) speculated that, 210 although these participants had been engaged in exercises intended to condition the lumbar extensors, 211 their lumbar extensors may have in fact been untrained relative to their other musculature. The large ESs 212 in our trained participants further suggests this may be the case. Steele et al. (2013) concluded in their 213 review of the specificity of exercises designed to condition the lumbar extensors that many exercises 214 suggested by Mayer et al. (2008) may be inferior to ILEX. Indeed it may be that the lumbar extensor 215 musculature may become deconditioned relative to other musculature due to the lack of conditioning 216 effect from many typical trunk extension based exercises or movements (Steele et al., 2014 a). Our CON 217 group in fact lost ILEX strength over the intervention period highlighting this possibility (though it is 
218 unclear as to why a decrease of comparable magnitude to both the 1ST and 3ST groups' increases was

219 found). It thus remains possible that set volume may impact upon strength gains in those previously 220 engaged in ILEX RT who thus have trained lumbar extensors. This is an area that requires further 221 investigation.

223 The practical implications of these findings are of importance. The typical recommendations for a single 224 set approach for ILEX has been noted as time efficient for addressing pain and disability in people with 225 chronic LBP (Steele et al., 2014 ). However, as noted, prior reviews have not offered recommendation 226 as to what set volume might be best for ILEX RT for the purposes of conditioning the lumbar extensors 227 (Steele et al., 2013), something of particular important for those wishing to reduce risk of low back injury 228 (Stone, 1990; Lauersen et al., 2013; Steele et al., 2014a ; 2014 ${ }^{\mathrm{b}}$ ). This study suggests that a single set 229 approach may be a time efficient method to effectively condition the lumbar extensors. Including the 230 warm-up set the single set group performed between 1.56 and 2.24 minutes of exercise whereas the 231 multiple set group, including between set rest periods, trained for between 9.48 and 11.12 minutes from 232 beginning exercise to end. Though this may appear a practically small difference, in the context of a 233 training program including a selection of other exercises that are performed using either single or 234 multiple sets this could add up to considerably more training time.

236 Limitations of the present study should be considered. Though our sample size was similar to other 237 research examining single and multiple set RT, due to drop outs, we fell slightly below the sample size 238 indicated by our power analysis (10 participants). However, calculations of observed power for group 239 effects upon ILEX strength changes were $\beta=0.966$ and thus this study can be considered as adequately 240 powered to detect between group differences. Despite the study being adequately powered the limitations 241 to practical conclusions should be noted. The study was only 6 weeks in duration and thus, although 
242 other studies of longer duration have suggested no difference, it remains the possibility that differences

243 between single and multiple set ILEX in trained participants might manifest over longer training 244 durations. Further, recent studies considering the effects of set volume have included greater than 3 sets 245 and suggested that both 5 (Radaelli et al., 2014a) and 8 sets (Marshall et al., 2011) may produce greater 246 strength adaptations. Whether higher set volumes may produce greater increases for ILEX RT remains 247 to be investigated.

249 Conclusion

250 To conclude, the results of this study suggest that strength changes resulting from an ILEX RT 251 intervention in recreationally trained males are unaffected by differing set volumes. We compared one 252 and three set training finding no difference in the magnitude of strength changes that occurred. Both 253 approaches appeared to significantly improve ILEX strength. Thus we can recommend that a single set 254 approach to ILEX RT is a time efficient and sufficient approach for the purposes of conditioning the 255 lumbar extensors. Indeed considering evidence that RT can be used for reducing injury risk this might 256 also represent a time efficient approach for primary prevention of low back injury and pain and future 257 research should look to investigate this potential.

\section{References}

1. Adnan MA, Kadir ZA, Yusof SM, Mazaulan M, Mohamed MAAR, 2014. Single versus two sets of resistance training on muscular endurance, strength and fat percentages among recreationally trained men. In: Adnan R, Ismail SI, Sulaiman N, eds. Proceedings of the International Colloquium on Sports Science, Exercise, Engineering and Technology 2014 (ICoSSEET 2014). London: Springer:249-258 
2. American College of Sports Medicine, 2009. Progression models in resistance training for healthy adults. Medicine and Science in Sports and Exercise; 41(3): 687-708

3. Bahr R, Andersen SO, Loken S, Fossan B, Hansen T, Holme I, 2004. Low back pain among endurance athletes with and without specific back loading - a cross sectional survey of cross country skiers, rowers, orienteerers, and nonathletic controls. Spine; 29(4): 449-454

4. Baker JS, Davies D, Cooper SM, Wong DP, Buchan DS, Kilgore L, 2013. Strength and body composition changes I recreationally strength-trained individuals: comparison of one versus three sets resistance-training programmes. BioMed Research International

5. Bono CM, 2004. Low back pain in athletes. Journal of Bone and Joint Surgery; 86(2): 382-396

6. Bruce-Low S, Smith D, Burnet S, Fisher J, Bissell G, Webster L, 2012. One lumbar extension training session per week is sufficient for gains and reductions in pain in patients with chronic low back pain ergonomics. Ergonomics; 55(4):500 - 507

7. Carpenter DM, Graves JE, Pollock ML, Leggett SH, Foster D, Holmes B, Fulton MN, 1991. Effect of 12 and 20 weeks of resistance training on lumbar extension torque production. Physical Therapy; 71(8): 580-588

8. Carpinelli RN, 2012. Critical review of a meta-analysis for the effect of single and multiple sets of resistance training on strength gains. Medicina Sportiva;16(3):122-130

9. Cohen J, 1992. A power primer. Psychological Bulletin;112:155-159

10. Correa CS, Teixeira BC, Bittencourt A, Lemos L, Marques NR, Radaelli R, Kruger RL, Reischak-Oliveira A, Pinto RS, 2014. Effects of high and low volume of strength training on muscle strength, muscle volume and lipid profile in postmenopausal women. Journal of Exercise Science and Fitness; 12(2):62-67

11. Fisher J, Steele J, Bruce-Low S, Smith D, 2011. Evidence based resistance training recommendations. Medicina Sportiva; 15(3): 147-162 
12. Frohlich M, Emrich E, Schmidtbleicher D, 2010. Outcome effects of single-set versus multipleset training - an advanced replication study. Research in Sports Medicine; 18:157-175

13. Graned H, Morelli B, 1988. Low back pain among retired wrestlers and heavyweight lifters. American Journal of Sports Medicine; 16(5): 530-533

14. Graves JE, Holmes BL, Leggett SH, Carpenter DM, Pollock ML, 1991. Single versus multiple set dynamic and isometric lumbar extension training. Proceedings of the XIth International Congress of the World Confederation for Physical Therapy; 1991, Jul 28 - Aug 2, 1340-1342

15. Graves JE, Pollock ML, Foster D, Leggett SH, Carpenter DM, Vuoso R, Jones A, 1990a. Effect of training frequency and specificity on isometric lumbar extension strength. Spine; 15(6): 504509

16. Graves JE, Pollock ML, Carpenter DM, Leggett SH, Jones A, MacMillan M, Fulton M, $1990^{\text {b }}$. Quantitative assessment of full range of motion isometric lumbar extension strength. Spine;15(4):289 - 294 24(1): $128-133$

17. Graves JE, Pollock ML, Leggett SH, Carpenter DM, Fix CK, Fulton MN, 1992. Limited rangeof-motion lumbar extension strength training. Medicine and Science in Sports and Exercise;

18. Hass CJ, Garzarella L, De Hoyos D, Pollock ML, 2000. Single versus multiple sets in long term recreational weightlifters. Medicine and Science in Sports and Exercise; 32(1):235-242

19. Inanami H, 1991. Iwai Orthopedic Hospital rehabilitation program. Paper presented at International Spinal Rehabilitation Update 1991 Symposium, Daytona; 1991

20. Kadir ZA, Nadzalan AM, Yusof SM, Aiman S, Shapie MNM, 2014. Single- versus three-set resistance training on strength and power among untrained men. In: Adnan R, Ismail SI, Sulaiman $\mathrm{N}$, eds. Proceedings of the International Colloquium on Sports Science, Exercise, Engineering and Technology 2014 (ICoSSEET 2014). London: Springer, 177-187 
21. Kraft DE, 2002. Low back pain in the adolescent athlete. Paediatric Clinics of North America;49(3): 643-653

22. Krieger JW, 2009. Single versus multiple sets of resistance exercise: a meta-regression. Journal of Strength and Conditioning Research;23(6):1890-1901

23. Lauersen JB, Bertelsen DM, Andersen LB, 2013. The effectiveness of exercise interventions to prevent sports injuries: a systematic review and meta-analysis of randomised controlled trials. British Journal of Sports Medicine; Online First

24. Marshall PWM, McEwen M, Robbins DW, 2011. Strength and neuromuscular adaptation following on, four, and eight sets of high intensity resistance exercise in trained males. European Journal of Applied Physiology;111:3007-3016

25. Mayer J, Mooney V, Dagenais S, 2008. Evidence informed management of chronic low back pain with lumbar extensor strengthening exercises. The Spine Journal; 8: 96-113

26. Medeiros HS Jr, Mello RS, Amorim MZ, Koch AJ, Machado M, 2013. Planned intensity reduction to maintain repetitions within recommended hypertrophy range. International Journal Sports Physiology and Performance;8(4): 384-390

27. National Institute for Health and Clinical Excellence, 2009. Low back pain: early management of persistent non-specific low back pain. London: Royal College of General Practitioners

28. Office for National Statistics, 2000. Social Trends 30. London: The Stationary Office

29. Office for National Statistics, 2010. Social Trends 40. Basingstoke: Palgrave Macmillan

30. Otto RM, Carpinelli RN, 2006. A critical analysis of the single versus multiple set debate. Journal of Exercise Physiology;9(1):32-57

31. Peterson MD, Rhea MR, Alvar BA, 2004. Maximizing strength development in athletes: a metaanalysis to determine the dose-response relationship. Journal of Strength and Conditioning Research;18(2):377-382 
32. Pollock ML, Graves JE, Leggett SH, Young WG, Gazzarella Z, Carpenter DM, 1991. Accuracy of counter weighting to account for upper body mass in testing lumbar extension strength. Medicine and Science in Sports and Exercise;23:66

33. Pollock ML, Leggett SH, Graves JE, Jones A, Fulton M, Cirulli J, 1989. Effect of resistance training on lumbar extension strength. American Journal of Sports Medicine; 17(5): 624-629

34. Radaelli R, Fleck SJ, Leite T, Leite RD, Pinto RS, Fernandes L, Simao R, 2014 a . Does respsonse of 1, 3 and 5 sets of resistance exercise on strength, local muscular endurance and hypertrophy. Journal of Strength and Conditioning Research; Epub ahead of print

35. Radaelli R, Botton, CE, Wilhelm EN, Bottaro M, Brown LE, Lacerda F, Gaya A, Moraes K, Peruzzolo A, Pinto RS, 2014 ${ }^{\mathrm{b}}$. Time course of low- and high-volume strength training on neuromuscular adaptations and muscle quality in older women. Age;36:881-892

36. Radaelli R, Wilhelm EN, Botton CE, Rech A, Bottaro M, Brown LE, Pinto RS, 2014c . Effects of single vs. multiple-set short-term strength training in elderly women. Age; 36(6):9720

37. Radaelli R, Wilhelm EN, Botton CE, Bottaro M, Cadore EL, Brown LE, Pinto RS, 2013a . Effect of two difference strength training volumes on muscle hypertrophy and quality in elderly women. Journal of Sports Medicine and Physical Fitness;53(Suppl 1-3):1-6

38. Radaelli R, Botton CE, Wilhelm EN, Bottaro M, Lacerda F, Gaya A, Moraes K, Peruzzolo A, Brown LE, Pinto RS, 2013 ${ }^{\text {. }}$ Low- and high-volume strength training induces similar neuromuscular improvements in muscle quality in elderly women. Experimental Gerontology;48(8):710-716

39. Rhea MR, Alvar BA, Burkett LN, Ball SD, 2003. A meta-analysis to determine the dose response for strength development. Medicine and Science in Sports and Exercise;35(3):456-464

40. Robinson ME, Greene AF, O’Connor P, Graves JE, MacMillan M, 1992. Reliability of lumbar isometric torque in patients with chronic low back pain. Physical Therapy;72(3):186 - 190 
41. Steele J, Bruce-Low S, Smith D, 2014․ A reappraisal of the deconditioning hypothesis in low back pain: review of evidence from a triumvirate of research methods on specific lumbar extensor deconditioning. Current Medical Research and Opinion; 30(5): 865-911

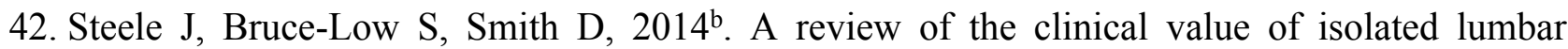
extension resistance training for chronic low back pain. $P M \& R$; Epub ahead of print

43. Steele J, Bruce-Low S, Smith D, 2013. A review of the specificity of exercises designed for conditioning the lumbar extensors. British Journal of Sports Medicine; Online First

44. Steele J, 2014. Intensity; in-ten-si-ty; noun. 1. Often used ambiguously within resistance training. 2. Is it time to drop the term altogether? British Journal of Sports Medicine;48:1859-1588

45. Stone MH, 1990. Muscle conditioning and muscle injuries. Medicine and Science in Sports and Exercise; 22(4): 457-462

46. Sward L, Hellstrom M, Jacobsson, Peterson L, 1990. Back pain and radiologic changes in the thoraco-lumbar spine of athletes. Spine; 15(2): 124-129

47. Tucci JT, Carpenter DM, Pollock ML, Graves JE, Leggett SH, 1992. Effect of reduced frequency of training and detraining on lumbar extension strength. Spine; 17(2): 1497-1501

48. Waddell G, Burton AK, 2000. Occupational health guidelines for the management of low back pain at work: evidence review. Occupational Medicine; 51(2): 126-135

49. Walker BF, 2000. The prevalence of low back pain: a systematic review of the literature from 1966 to 1998. Journal of Spinal Disorders; 13(3), pp $205-217$

50. Whitley E, Ball J, 2002. Statistics review 4: sample size calculations. Critical Care;6:335-341

51. Winett RA, 2004. Meta-analyses do not support performance of multiple sets or high volume resistance training. Journal of Exercise Physiology;7(5):10-20

52. Wolfe BL, LeMura LM, Cole PJ, 2004. Quantitative analysis of single- vs multiple-set programs in resistance training. Journal of Strength and Conditioning Research;18(1):35-47 
53. World Health Organisation, 1998. The World Health Report 1998: Life in the $21^{\text {st }}$ century: A vision for all. Geneva: Office of Publications, World Health Organisation, 


\section{Table $\mathbf{1}$ (on next page)}

Participant demographic characteristics 
3 Table 1. Participant demographic characteristics

\begin{tabular}{lccc}
\hline & $1 \mathrm{ST}$ & $3 \mathrm{ST}$ & CON \\
\hline Participants (No.) & 9 & 8 & 9 \\
Age (years) & $21 \pm 2$ & $21 \pm 1$ & $20 \pm 1$ \\
Stature (cm) & $174.7 \pm 7.6$ & $178.5 \pm 8.7$ & $178.4 \pm 6.3$ \\
Body Mass (kg) & $74.3 \pm 9.1$ & $78.7 \pm 10.3$ & $3 \pm .7 \pm 7.8$ \\
Training Experience (years) & $3 \pm 1$ & $5 \pm 2$ & $339.84 \pm 64.82$ \\
Baseline ILEX Strength (Nm) & $277.66 \pm 99.99$ & $335.71 \pm 80.04$ & 72 \\
ILEX Range of Motion $\left(^{\circ}\right)$ & 72 & 72 & \\
\end{tabular}

4

5 
Figure 1 (on next page)

CONSORT diagram 


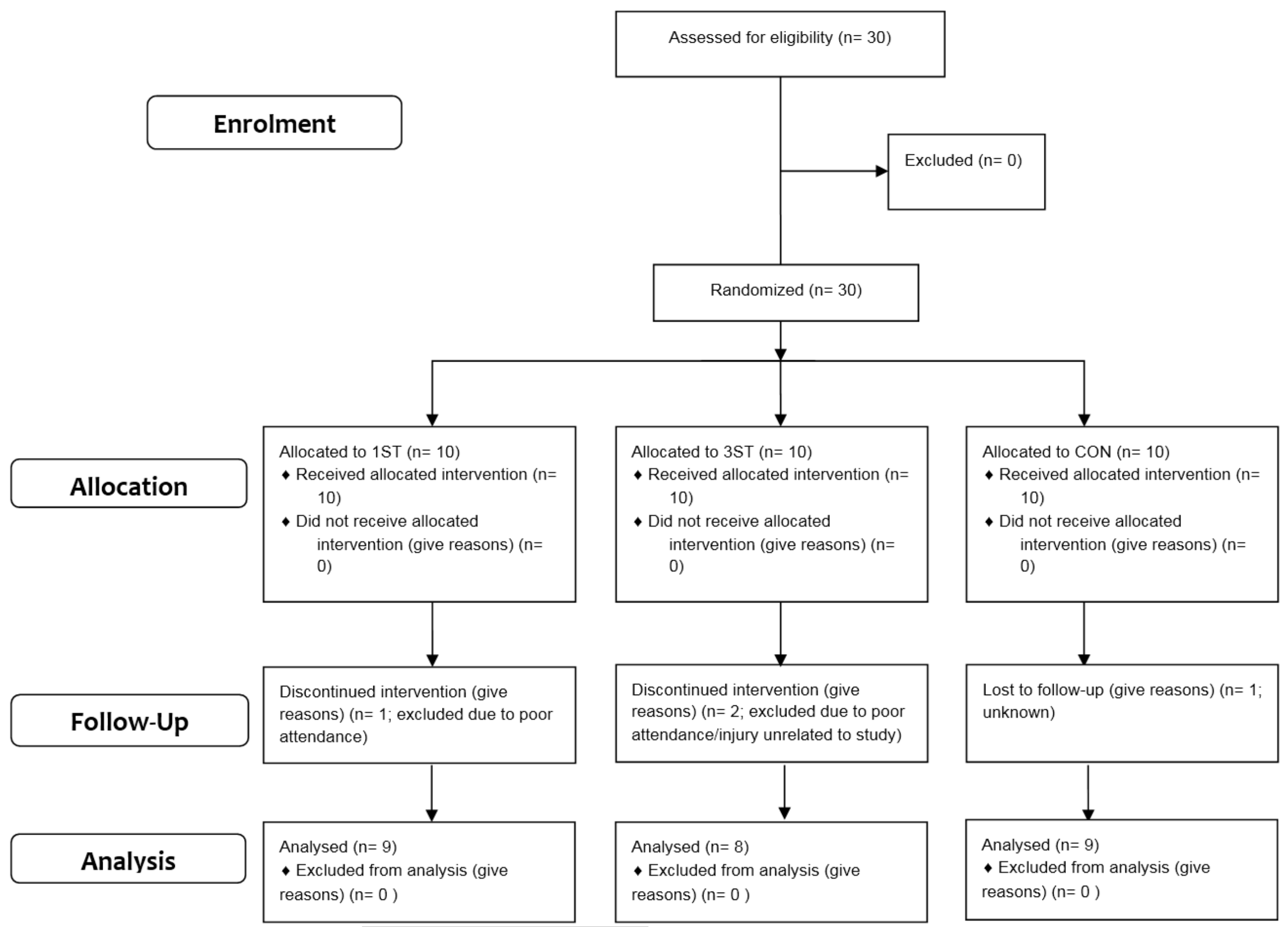


Figure 2 (on next page)

Mean change in ILEX strength with $95 \% \mathrm{Cls}$ 
PeerJ Reviewing Manuscript

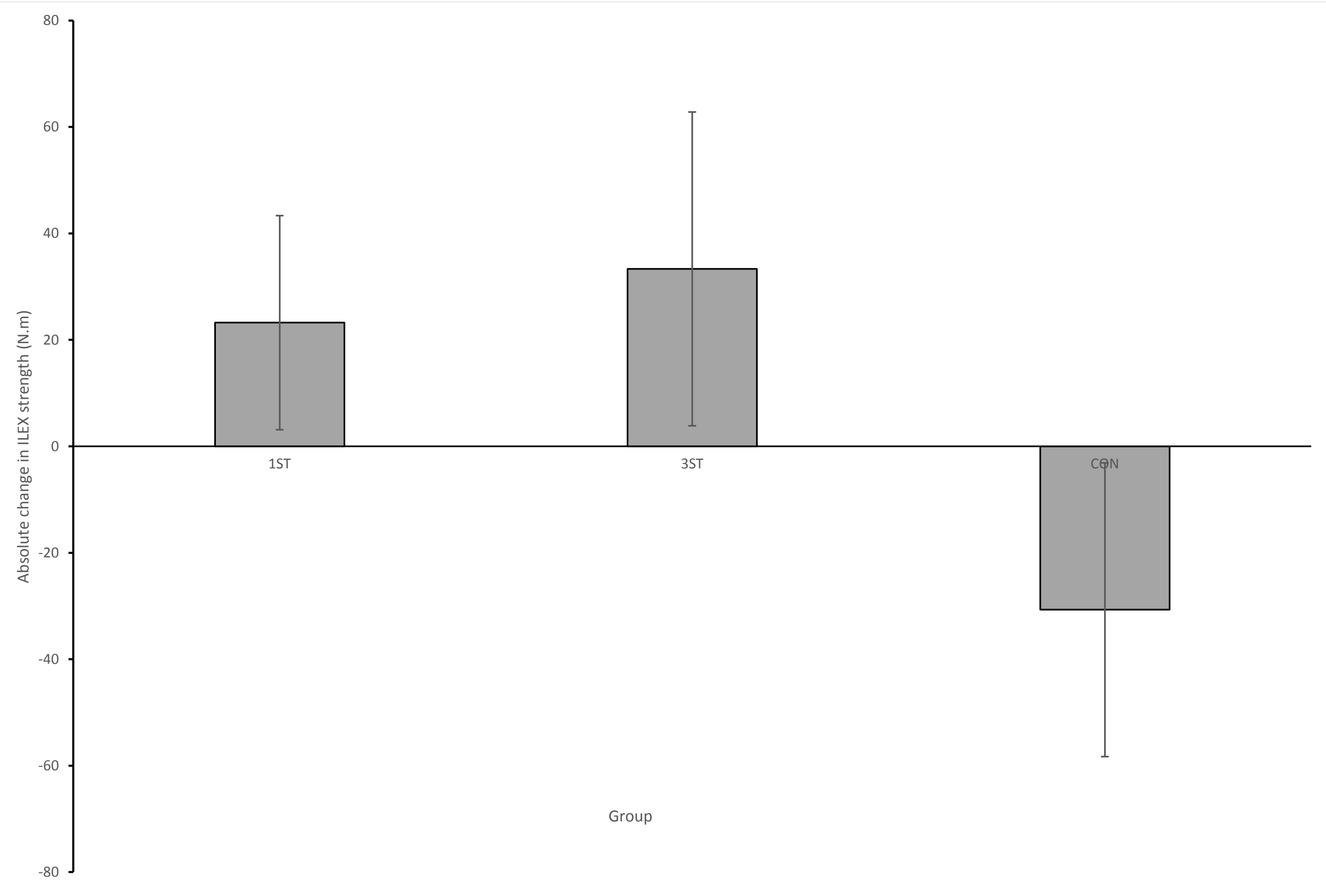

